\title{
Nutritional predictors of mortality in prevalent peritoneal dialysis patients
}

\author{
Sylwia Malgorzewicz ${ }^{\bowtie}$, Michal Chmielewski², Malgorzata Kaczkan¹, Paulina Borek², \\ Monika Lichodziejewska-Niemierko ${ }^{2,3}$ and Boleslaw Rutkowski² \\ 1Department of Clinical Nutrition; ${ }^{2}$ Department of Nephrology, Transplantology and Internal Medicine; Department of Palliative Medicine; \\ Medical University of Gdańsk, Gdańsk, Poland
}

\begin{abstract}
Malnutrition remains one of the major predictors of mortality in peritoneal dialysis (PD) patients. The aim of the study was to evaluate the nutritional status of prevalent PD patients, and to determine the best predictors of outcome among anthropometric and laboratory indices of nutrition. The study included 106 prevalent PD patients from a single university-based unit. Anthropometric assessment at baseline included: body mass, body mass index (BMI), skinfold thickness, lean body mass (LBM), content of body fat $(\% \mathrm{~F})$, mid-arm muscle circumference (MAMC). Laboratory analysis comprised of albumin and total cholesterol. Additionally, each patient underwent a subjective global assessment (SGA). The patients were followed for 36 months. Survival analyses were made with the Kaplan-Meier survival curve and the Cox proportional hazard model. Following SGA, malnutrition was diagnosed in $30(28 \%)$ patients. Importantly, eight of the malnourished patients $(27 \%)$ were nevertheless overweight or obese. Body weight and BMI showed complete lack of association with the outcome. In KaplanMeier analysis low: LBM, MAMC, albumin and cholesterol were significantly related to mortality. Cox analysis revealed that, following adjustment, LBM below median was independently associated with poor outcome (hazard ratio [HR] 3.15, 95\% confidence interval [CI] 1.17$8.49, p=0.02$ ). Moreover, the lowest quartile of total cholesterol showed independent association with mortality (HR 8.68, Cl 2.14-35.21, $p<0.01$ ). Malnutrition is prevalent in patients undergoing PD, and overweight/obesity does not preclude its appearance. The most valuable nutritional indices in predicting outcome in this cohort were LBM and total cholesterol concentration.
\end{abstract}

Key words: peritoneal dialysis, nutritional status, mortality

Received: 01 June, 2015; revised: 18 November, 2015; accepted: 06 January, 2016; available on-line: 27 January, 2016

\section{INTRODUCTION}

Malnutrition or, according to the current nomenclature, protein-energy wasting (PEW) is highly prevalent among dialysis subjects (Fouque et al., 2008). Uremic toxaemia, bioincompatibility of dialysis membranes in haemodialysis (HD) and of dialysis fluids in peritoneal dialysis (PD), low-grade inflammation, oxidative stress, co-morbidities are among the major culprits responsible for PEW in dialysis-dependant patients. On the other hand, the prevalence of overweight and obesity is growing worldwide, both in the general population and in di- alysis patients. The risk of obesity in PD subjects is additionally increased by glucose-based dialysis fluids.

Although both HD and PD have been established and successful therapies for the treatment of patients with end-stage renal disease (ESRD) have been developed, the mortality rate of dialysis subjects remains unacceptably high. Apart from the traditional risk factors, there are the specific non-traditional ones, such as inflammation, oxidative stress, and endothelial dysfunction that seem to be the major culprits (Malgorzewicz et al., 2010). As stated above, they are also strongly associated with PEW.

To reduce this enormous risk of death, the risk factors contributing to mortality should be identified and controlled. Previous studies have documented strong association between indices of PEW and morbidity/mortality of HD subjects (Bergstrom et al., 1995; Burowes et al., 2005; Carrero et al., 2008). Similar relationships have also been found in PD patients (Churchill et al., 1996). However, the issue which of the commonly used markers of nutrition is the strongest risk factor for poor outcome in dialysis patients remains opened Moreover, although in HD population obesity seems to associate with survival benefit, the data on this relationship in PD patients is inconclusive (McDonald et al., 2003; Snyder et al., 2003; Park et al., 2014).

Therefore, the aim of the study was to evaluate the nutritional status of prevalent PD patients, and to determine the best predictors of outcome among anthropometric and laboratory indices of nutrition.

\section{METHODS}

Patients and anthropometric evaluation. The study population consisted of 106 prevalent PD patients from a single nephrology centre, treated with PD for at least three months. The recruitment took place in 2006-2008. The study exclusion criteria were: age below 18 years, or unwillingness to participate. Informed consent was obtained from each patient. The local ethics committee approved the study protocol.

The 7-points subjective global assessment (SGA) was used to evaluate the overall protein-energy nutritional

e-mail: sylwia@tetra.pl

Abbreviations: BMI, body mass index; $\mathrm{Cl}$, confidence interval; ESRD, end-stage renal disease; FM, fat mass; \%F, content of body fat; HD, haemodialysis; HR, hazard ratio; LBM, lean body mass; MAMC, mid-arm muscle circumference; NCEP ATP III, National Cholesterol Education Program Adult Treatment Panel III; PD, peritoneal dialysis; PEW, protein-energy wasting; SGA, subjective global assessment 
status, and patients presenting SGA score of 1-2 points were classified as malnourished (NCEP, 2002). The assessment was performed by a single experienced dietician. Body mass index (BMI) was calculated as weight in $\mathrm{kg}$ per (height in $\mathrm{m})^{2}$. Body fat $(\% \mathrm{~F})$ and lean body mass (LBM) were determined with the near-infrared technology (Futrex 2000A; Futrex Inc., USA). The triceps skinfold thickness was assessed using a Holtain calliper (Holtain LTD, Crymych U.K); mid-arm muscle circumference (MAMC) was measured with a tape measure.

Serum albumin, total cholesterol concentration, and other biochemical measures were assessed with routine laboratory methods. Dialysis adequacy was estimated with a weekly $\mathrm{Kt} / \mathrm{V}$. Co-morbidities were defined as the presence of diabetes mellitus and/or cardiovascular disease.

Statistical analysis. Results are expressed as mean and standard deviation or median and interquartile range, as appropriate. A $p$-value $<0.05$ was considered to be statistically significant. Comparisons between two groups were assessed for continuous variables with Student's unpaired t-test, or Mann-Whitney test, as appropriate. As many values were not normally distributed, Spearman's rank correlation was used to determine correlations between nutrition indices.

Survival analyses were made with the Kaplan-Meier survival curve or the Cox proportional hazard model. Survival was determined from the day of examination, with a median follow-up period of 18 (range 1-36) months. Patients were censored at transplantation, transfer to HD or upon completing the follow-up period, without loss of follow-up of any patient. The relative risk of mortality was determined by multivariate Cox regression analysis and presented as hazard ratio (HR); $95 \%$ confidence intervals (CI)]. The statistical analysis was performed using statistical software Statistica version 7.1 (StatSoft Inc.).

\section{RESULTS}

The study population consisted of 106 patients (56 males; $53 \%$ ), with a median age of 56 years (range 28-79 years). The patients had undergone dialysis for a median of 11 months (range 3-87 months). The primary kidney diseases were: diabetic nephropathy $(24.5 \%)$, primary

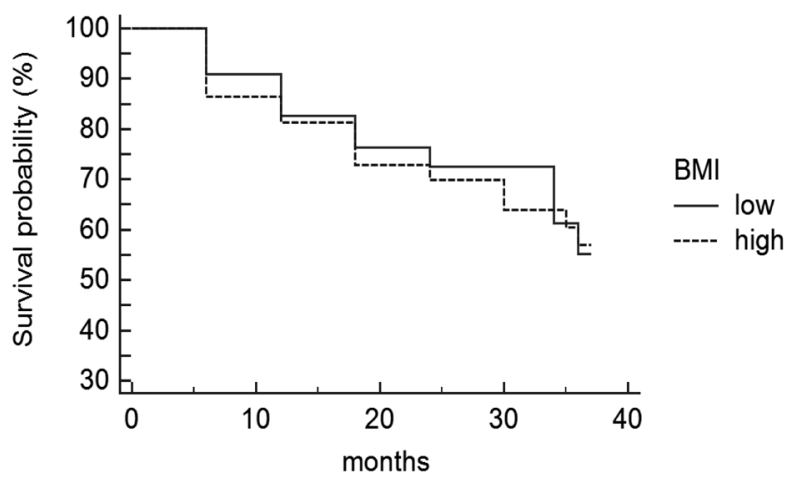

Figure 1. Mortality risk in patients undergoing PD according to their body mass index (BMI); low BMI was defined as the value lower than the median $\left(24.6 \mathrm{~kg} / \mathrm{m}^{2}\right)$.

glomerulonephritis (21.2\%), hypertensive nephropathy $(18.8 \%)$, reflux nephropathy $(10.2 \%)$, chronic pyelonephritis $(8.6 \%)$, and other or unknown $(16.7 \%)$. The type of the PD therapy engaged was: continuous ambulatory (CAPD) in $75.0 \%$, and automated (APD) in $25.0 \%$. Out of the total cohort, 30 patients $(28 \%)$ were treated with lipid-lowering medications.

When assessed on the basis of SGA, PEW was present in $30(28 \%)$ patients. The clinical characteristics of malnourished subjects, as compared to the well-nourished group, are presented in Table 1. As displayed in the Table, the subjects with malnutrition were characterized by significantly lower BMI in comparison to the rest of the studied group. However, it has to be stated that increased BMI did not preclude PEW, as eight of the 30 malnourished patients $(27 \%)$ were, nevertheless, overweight or obese.

During the three years of follow-up, 29 (27\%) patients died, $34(32 \%)$ underwent kidney transplantation, and nine $(8 \%)$ were transferred to HD. Those who died during the follow-up were older, with a median age of 71.0 (60.8-74.0) vs. 53.0 (43.5-63.0) years $(p<0.001)$. This group had also a higher proportion of patients with malnutrition $(38 \%$ vs. $24 \%, p=0.26)$, although this difference did not reach statistical significance. Finally, these patients were characterized by a significantly lower albumin concentration $(35.5 \pm 4.6$ vs. $39.1 \pm 4.5 \mathrm{mg} / \mathrm{L}$,

Table 1. The general characteristics of malnourished vs. well-nourished PD patients; BMI, body mass index; TC, total cholesterol; LBM, lean body mass; \%F, content of body fat.

\begin{tabular}{llcc}
\hline & Malnourished $(\mathrm{N}=30)$ & Well-nourished (N=76) & $p$-value \\
\hline Age (years) & $57.5 \pm 16.5$ & $55.6 \pm 12.8$ & 0.2 \\
\hline Albumin (mg/l) & $37.5 \pm 5.4$ & $38.3 \pm 4.6$ & 0.2 \\
\hline Gender (\% males) & 60 & 50 & 0.07 \\
\hline BMl & $23.7 \pm 4.4$ & $26.1 \pm 3.7$ & $<0.005$ \\
\hline TC (mg/dl) & $232 \pm 47$ & $231 \pm 71$ & 0.9 \\
\hline Dialysis vintage (months) & $7.0(3.0-23.3)$ & $12.0(3.3-31.5)$ & 0.6 \\
\hline LBM (kg) & $50.3 \pm 8.6$ & $52.5 \pm 6.6$ & 0.09 \\
\hline \%F & $26.8 \pm 6.0$ & $26.6 \pm 4.5$ & 0.9 \\
\hline Chronic co-morbidities (\%) & 58 & 51 & 0.4 \\
\hline Kt/V & $2.09 \pm 0.18$ & $2.26 \pm 0.36$ & 0.02 \\
\hline
\end{tabular}




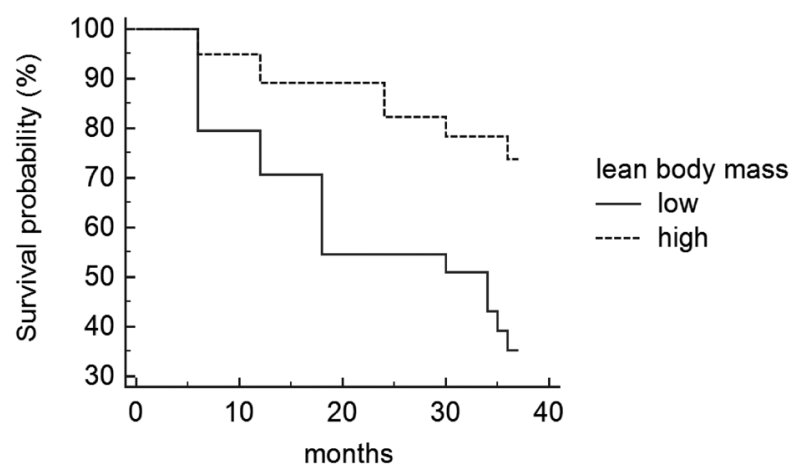

Figure 2. Mortality risk in patients undergoing PD according to their lean body mass (LBM); low LBM was defined as the value lower than the median $(51.4 \mathrm{~kg})$.

$p<0.001)$, and worse $\mathrm{Kt} / \mathrm{V}(2.12 \pm 0.22$ vs. $2.25 \pm 0.35$, $p=0.03)$, as compared to survivors. The differences in total cholesterol (212 \pm 82 vs. $239 \pm 57 \mathrm{mg} / \mathrm{dl}, p=0.07)$, and LBM $(50.3 \pm 5.4$ vs. $52.6 \pm 7.9 \mathrm{~kg}, p=0.08)$ were of marginal significance. There were no differences in BMI, $\% \mathrm{~F}$ or MAMC between the patients who died and the survivors.

Malnutrition, as assessed by SGA, showed a marginal association with poor outcome, when assessed with the Kaplan-Meier analysis (rho 3.1, $p=0.07$ ). It further lost its significance in predicting mortality following adjustment for: age, gender, inflammation and co-morbidities. Although BMI was related to malnutrition, it showed absolutely no association with the risk of death, both in univariate and multivariate survival analyses (Fig. 1).

Decreased albumin, total cholesterol and low MAMC were significantly associated with poor outcome. However, these relationships were lost after adjustment for potential confounders, except for the cholesterol concentration. Patients belonging to the lowest quartile of the total cholesterol level $(<199$ $\mathrm{mg} / \mathrm{dl}$ ) had a significantly worse survival (rho 6.5, $p=0.01$ ), when compared to the rest of the studied group. This association persisted despite adjustment for potential confounders: age, gender, dialysis vintage and adequacy, residual diuresis, co-morbidities, albumin, CRP and the use of lipid-lowering medication (HR 7.29, CI 1.85-28.80, $p<0.01$ ).

Among anthropometric measures, low LBM turned out to be the strongest predictor of mortality, both as a crude LBM and as normalized to body weight (Fig. 2). Cox proportional hazards analysis revealed that subjects having LBM below the median $(<51.4 \mathrm{~kg})$ were exposed to significantly increased risk of death (Table 2), as compared to the rest of the patients. It is noteworthy that LBM and BMI were closely related $(r=0.32, p<0.005)$. Nevertheless, their usefulness in predicting the outcome was entirely different in the studied population.

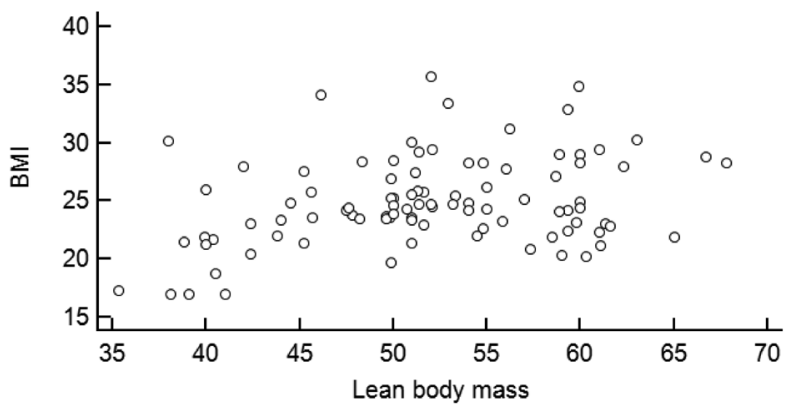

Figure 3. Spearman rank correlation between lean body mass (LBM) and body mass index (BMI) in patients undergoing PD; $r=0.32, p<0.005$

\section{DISCUSSION}

In the present study, we demonstrated a high prevalence of PEW among PD patients, common also in overweight/obese subjects. Moreover, although various indices of PEW showed association with survival, only low cholesterol and LBM proved to be strong, independent risk factors for mortality in this patient population.

The high prevalence of malnutrition is a constant feature of end-stage renal disease (ESRD). Data from surveys conducted in dialysis subjects indicate that $1875 \%$ of this patient population show signs of PEW, the differences depending mainly on the assessment methods used (Fouque et al., 2008). The chronic uremic intoxication is among the major culprits, leading, on one hand, to decreased energy intake, and, on the other, to enhanced protein catabolism (Stenvinkel et al., 2000). Other potentially responsible factors include: co-morbidities, bioincopatibility of dialysis fluids, oxidative stress, depression, decreased physical activity etc. The PD population is a specific group of patients who are at risk of increasing weight, due to the constant glucose load from the dialysis fluids, but, on the other hand, at risk of wasting, due to continuous protein loss to the peritoneal cavity in addition to the abovementioned factors associated with uraemia. Our results clearly underlined this paradox, as almost one-third of the malnourished patients was, nevertheless, overweight or obese. This is in accordance with our previous studies that showed a similar incidence of malnutrition in both lean and overweight PD patients (Malgorzewicz et al., 2004).

In the present study, PEW, as assessed by SGA, turned out to be a weak predictor of mortality. It lost its significance when adjusted for potential confounders, especially the presence of inflammation and co-morbidities. This stays in accordance with previous findings in PD subjects (Chung et al., 2003). It might also be that SGA is not perfectly suited for diagnosing PEW. In the present study, the 7-point SGA was implemented to di-

Table 2. Crude and adjusted overall hazard ratios (and 95\% confidence intervals) of the low LBM on all-cause mortality ( $n=106)$

\begin{tabular}{|c|c|c|c|c|}
\hline & \multirow{2}{*}{ Hazard ratio } & \multicolumn{2}{|c|}{$95 \% \mathrm{Cl}$} & \multirow{2}{*}{$p$-value } \\
\hline & & lower & upper & \\
\hline Crude & 3.29 & 1.46 & 7.41 & 0.002 \\
\hline Adjusted for age and gender & 3.44 & 1.50 & 7.88 & 0.004 \\
\hline Adjusted for the above + dialysis vintage, adequacy, and residual diuresis & 3.55 & 1.30 & 9.69 & 0.01 \\
\hline Adjusted for the above + co-morbidities and inflammation & 3.24 & 1.18 & 9.89 & 0.02 \\
\hline
\end{tabular}


agnose PEW. This is an acknowledged, cheap, and easyto-perform tool to distinguish the wasted patients from the well-nourished ones. However, it has some limitation due to its poor inter-rater reliability (Tennankore et al., 2002). Therefore, in our study, all assessments were performed by a single experienced dietician.

In the studied population, there was no association between BMI and mortality. In the general population, obesity is an acknowledged risk factor for poor outcome (Haffner et al., 2007). In contrast, numerous studies performed in HD subjects showed the opposite association, with low BMI being the risk predictor (Park et al., 2014). The most common explanations for this risk factor paradox include: confounding by PEW, reverse causation and survival bias (Park et al., 2014). The relationship between BMI and outcome in PD seems even more confusing, as studies on this topic bring conflicting results. While some demonstrated a reverse association, similar to the one observed in HD patients (Visser et al., 1999), other showed that obesity is an independent risk factor for mortality, just as in the general population (McDolnad et al., 2003). Because body mass is composed of the fat mass and the fat-free, i.e. lean mass, BMI is affected by both of them. In the PD patients, BMI is also influenced by the hydration status. This probably explains, at least to some extent, the discrepant results of the abovementioned studies, and proves that BMI should not be regarded as a reliable marker of nutrition in this patient population.

In contrast, LBM turned out as a strong and independent predictor of outcome. This is in accordance with previous studies in which low LBM, estimated using creatinine index corrected with body weight, was an independent predictor of mortality in incident (Chung et al., 2000; Trivedi et al., 2005) and prevalent PD patients (Huang et al., 2013). The predictive value of LBM in HD patients seems much weaker (Noori et al., 2010). In this patient population, it is low fat mass (FM) that appears to be a risk factor (Noori et al., 2010). Currently, these discrepancies in the predictive utilities of FM and LBM between the two dialysis methods are difficult to explain. Both LBM and FM are measures of nutritional status. However, while LBM indicates muscle mass, FM is more a reflection of energy storage. In the present study, as in previous evaluations, it is LBM that showed association with survival, FM displaying no relationships at all.

The analysis of body composition was carried out with the near-infrared technology. Bioelectric impedance analysis is relatively accessible method of the body composition assessment. Obviously, it has to be remembered that it is potentially influenced by total hydration status, limiting its accuracy in states of volume overload (Tennankore et al., 2013). In the present study, albumin turned out to predict mortality only in an unadjusted analysis. It has to be stated that the role of albumin as a nutrition marker has been questioned. As it is a negative acute-phase reactant, its levels tend to decrease in the course of inflammation, which is a common complication of ESRD. Moreover, peritoneal albumin loss and hypervolemia can decrease its concentration. Indeed, in multivariate analysis, when inflammation and co-morbidities were included, albumin lost its predictive potential.

In contrast, in the present evaluation low cholesterol turned out to be a strong and independent risk factor for mortality. This is in accordance with previous studies in HD patients (Lowrie et al., 1990; Kilpatrick et al., 2007; Chmielewski et al., 2011). Moreover, similarly to these studies, hypercholesterolemia appeared protective. In the present evaluation cholesterol concentration above $200 \mathrm{mg} / \mathrm{dl}$ proved beneficial in the Cox analysis. The apparent association between low cholesterol and mortality is most commonly explained by the presence of malnutrition and/or inflammation. Indeed, some studies in HD and PD subjects seem to confirm this hypothesis (Liu et al., 2004; Habib et al., 2006). However, most evaluations that took malnutrition and inflammation into account in multivariate analyses demonstrated that low cholesterol concentration remains as an independent risk factor for poor outcome, while hyperlipidaemia seems beneficial (Chmielewski et al., 2009; Chmielewski et al., 2011; Kilpatrick et al., 2013). Moreover, Mendelian randomization studies in HD patients demonstrated that the relationship between hypocholesterolemia and mortality might be causal (Chmielewski et al., 2011). Similarly, experimental studies showing that lipoproteins are capable of binding bacterial endotoxins pointed to the possibility that in certain chronic disease states, as ESRD, low cholesterol might actually be harmful (Rauchhaus et al., 2000). However, it has to be stated that most of the studied patients did not have a severe hypocholesterolemia. The lowest quartile included patients with total cholesterol concentration lower than $199 \mathrm{mg} / \mathrm{dl}$. This is the target cholesterol level in the general population, defined as 'desirable' by the National Cholesterol Education Program Adult Treatment Panel III (NCEP ATP III) (NCEP, 2002). Still, such cholesterol concentration was associated with death in the studied group of PD patients.

In the dialysis population the worse prognosis is associated, among others, with longer duration of the dialysis treatment. In the studied group dialysis vintage was relatively short (median was 11 months), but already during the first three years of PD therapy, patients showed signs of malnutrition and statistical analysis demonstrated that low cholesterol and LBM are risk factors for mortality in this period.

\section{CONCLUSIONS}

Malnutrition is highly prevalent in patients undergoing $\mathrm{PD}$, and the presence of overweight/obesity does not preclude its appearance. It ought to be diagnosed and treated as it is associated with increased risk of death. Among the various indices of malnutrition, it is decreased cholesterol and LBM that proved to be strong and independent risk factors for mortality in this patient population.

\section{REFERENCES}

Bergstrom J (1995) Nutrition and mortality in hemodialysis. J Am Soc Nephrol 6: 1329-1341.

Burrowes JD, Dalton S, Backstrand J, Levin NW (2005) Patients receiving maintenance hemodialysis with low vs high levels of nutritional risk have decreased morbidity. I Am Diet Assoc 105: 563-572.

Carrero JJ, Chmielewski M, Axelsson J, Snaedal S, Heimburger O, Barany P, Suliman ME, Lindholm B, Stenvinkel P, Qureshi AR (2008) Muscle atrophy, inflammation and clinical outcome in incident and prevalent dialysis patients. Clin Nutr 27: 557-564. doi: 10.1016/j. clnu.2008.04.007.

Chmielewski M, Carrero JJ, Qureshi AR, Axelsson J, Heimburger O, Berglund L, Barany P, Rutkowski B, Lindholm B, Stenvinkel $\mathrm{P}$ (2009) Temporal discrepancies in the association between the apoB/apoA-I ratio and mortality in incident dialysis patients. I Intern Med 265: 708-716. doi: 10.1111/j.1365-2796.2009.02074.x.

Chmielewski M, Verduijn M, Drechsler C, Lindholm B, Stenvinkel P, Rutkowski B, Boeschoten EW, Krediet RT, Dekker FW (2011) Low cholesterol in dialysis patients - causal factor for mortality or an effect of confounding? Nephrol Dial Transplant 26: 3325-3331. doi: $10.1093 / \mathrm{ndt} / \mathrm{gfr} 008$. 
Chung SH, Lindholm B, Lee HB (2000) Influence of initial nutritional status on continuous ambulatory peritoneal dialysis patient survival. Perit Dial Int 20: 19-26.

Chung SH, Lindholm B, Lee HB (2003) Is malnutrition an independent predictor of mortality in peritoneal dialysis patients? Nephrol Dial Transplant 18: 2134-2140.

Churchill D, Taylor W, Keshoniah P (1996) Adequacy of dialysis and nutrition in continuous peritoneal dialysis: association with clinical outcomes. Canada-USA (CANUSA) Peritoneal Dialysis Study Group. J Am Soc Nephrol 7: 198-207.

Fouque D, Kalantar-Zadeh K, Kopple J, Cano N, Chauveau P, Cuppari L, Franch H, Guarnieri G, Ikizler TA, Kaysen G, Lindholm B, Massy Z, Mitch W, Pineda E, Stenvinkel P, Trevino-Becerra A, Wanner C (2008) A proposed nomenclature and diagnostic criteria for protein-energy wasting in acute and chronic kidney disease. Kidney Int 73: 391-398.

Habib AN, Baird BC, Leypoldt JK, Cheung AK, Goldfarb-Rumyantzev AS (2006) The association of lipid levels with mortality in patients on chronic peritoneal dialysis. Nephrol Dial Transplant 21: 2881-2892.

Haffner SM (2007) Abdominal adiposity and cardiometabolic risk: do we have all the answers? Am J Med 120: 10-16.

Huang JW, Lien YC, Wu HY, Yen CJ, Pan CC, Hung TW, Su CT, Chiang CK, Cheng HT, Hung KY (2013) Lean body mass predicts long-term survival in Chinese patients on peritoneal dialysis. PLoS One 8: e54976.

Kilpatrick RD, McAllister CJ, Kovesdy CP, Derose SF, Kopple JD, Kalantar-Zadeh K (2007) Association between serum lipids and survival in hemodialysis patients and impact of race. $J$ Am Soc Nephrol 18: 293-303

Liu Y, Coresh J, Eustace JA, Longenecker JC, Jaar B, Fink NE, Tracy RP, Powe NR, Klag MJ (2004) Association between cholesterol level and mortality in dialysis patients: role of inflammation and malnutrition. $J A M A$ 291: 451-459.

Lowrie EG, Lew NL (1990) Death risk in hemodialysis patients: the predictive value of commonly measured variables and an evaluation of death rate differences between facilities. Am J Kidney Dis 15: $458-482$.

Malgorzewicz S, Lichodziejewska-Niemierko M, Aleksandrowicz-Wrona E, Swietlik D, Rutkowski B, Lysiak-Szydlowska W (2010) Adipokines, endothelial dysfunction and nutritional status in peritoneal dialysis patients. Scand J Urol Nephrol 44: 445-451.
Malgorzewicz S, Lichodziejewska-Niemierko M, Rutkowski B, LysiakSzydlowska W (2004) Nutritional status and oxidative processes in diabetic and nondiabetic peritoneal dialysis patients. J Ren Nutr 14: 242-247.

McDonald SP, Collins JF, Johnson DW (2003) Obesity is associated with worse peritoneal dialysis outcomes in the Australia and New Zealand patient populations. J Am Soc Nephrol 14: 2894-2901.

NCEP — Third Report of the national cholesterol education program (NCEP) expert panel on detection, evaluation, and treatment of high blood cholesterol in adults (adult treatment panel III) Final Report. (2002) Circulation 106: 3143-3421.

Noori N, Kovesdy CP, Dukkipati R, Kim Y, Duong U, Bross R, Oreopoulos A, Luna A, Benner D, Kopple JD, Kalantar-Zadeh $K$ (2010) Survival predictability of lean and fat mass in men and women undergoing maintenance hemodialysis. Am J Clin Nutr 92: 1060-1070. doi: 10.3945/ajcn.2010.29188.

Park J, Ahmadi SF, Streja E, Molnar MZ, Flegal KM, Gillen D, Kovesdy CP, Kalantar-Zadeh K (2014) Obesity paradox in endstage kidney disease patients. Prog Cardiovasc Dis 56: 415-425. doi: 10.1016/j.pcad.2013.10.005.

Rauchhaus M, Coats AJ, Anker SD (2000) The endotoxin-lipoprotein hypothesis. Lancet 356: 930-933.

Snyder JJ, Foley RN, Gilbertson DT, Vonesh EF, Collins AJ (2003) Body size and outcomes on peritoneal dialysis in the United States. Kidney Int 64: 1838-1844.

Stenvinkel P, Heimburger O, Lindholm B, Kaysen GA, Bergstrom J (2000) Are there two types of malnutrition in chronic renal failure? Evidence for relationships between malnutrition, inflammation and atherosclerosis (MIA syndrome). Nephrol Dial Transplant 15: 953-960.

Tennankore KK, Bargman JM (2013) Nutrition and the kidney: recommendations for peritoneal dialysis. Adv Chronic Kidney Dis 20: 190201. doi: 10.1053/j.ackd.2012.10.010.

Trivedi H, Tan SH, Prowant B, Sherman A, Voinescu CG, Atalla J, Khanna R, Nolph K (2005) Predictors of death in patients on peritoneal dialysis: the Missouri Peritoneal Dialysis Study. Am J Nephrol 25: 466-473.

Visser R, Dekker FW, Boeschoten EW, Stevens P, Krediet RT (1999) Reliability of the 7-point subjective global assessment scale in assessing nutritional status of dialysis patients. Adv Perit Dial 15: 222225 . 